\title{
NMDA Receptor Gating of Information Flow through the Striatum In Vivo
}

\author{
Pablo E. Pomata, Mariano A. Belluscio, Luis A. Riquelme, and M. Gustavo Murer \\ Laboratorio de Fisiología de Circuitos Neuronales, Departamento de Fisiología y Biofísica, Facultad de Medicina, Universidad de Buenos Aires, Ciudad de \\ Buenos Aires (CP1121), Argentina
}

\begin{abstract}
A role of NMDA receptors in corticostriatal synaptic plasticity is widely acknowledged. However, the conditions that allow NMDA receptor activation in the striatum in vivo remain obscure. Here we show that NMDA receptors contribute to sustain the membrane potential of striatal medium spiny projection neurons close to threshold during spontaneous UP states in vivo. Moreover, we found that the blockade of striatal NMDA receptors reduces markedly the spontaneous firing of ensembles of medium spiny neurons during slow waves in urethane-anesthetized rats. We speculate that recurrent activation of NMDA receptors during UP states allows off-line information flow through the striatum and system level consolidation during habit formation.
\end{abstract}

Key words: NMDA receptor; striatum; frontal cortex; slow waves; UP states; basal ganglia

\section{Introduction}

NMDA channel opening requires concurrent postsynaptic depolarization and glutamate binding. This coincident detection ability presumably underlies NMDA receptor (NMDAR) involvement in learning. Recent studies suggest that, in addition to online activation during learning, off-line NMDAR reactivation could be necessary for proper memory consolidation and storage (Wang et al., 2006). Moreover, NMDARs may play a more basic role in shaping spontaneous activity or information flow in the brain (Daw et al., 1993; Schiller and Schiller, 2001). Nevertheless, the conditions that allow NMDAR activation in the striatum in vivo remain unclear.

The striatum is the main recipient of cortical inputs to the basal ganglia. Its medium spiny projection neurons (MSNs), which constitute $90 \%$ of the striatal neurons, relay salient cortical inputs to basal ganglia output. This flow of information is likely involved in the initiation and sequencing of learned actions (Graybiel, 1998) and in habit formation through NMDARdependent corticostriatal plasticity (Kelley et al., 2003; Pawlak and Kerr, 2008). Action potentials in MSNs only occur during plateau depolarizations called UP states, which are induced by excitatory input during waves of cortical firing (Mahon et al., 2001; Tseng et al., 2001; Kasanetz et al., 2006). Thus, UP states enable information flow through the striatum (Wilson, 1993;

\footnotetext{
Received Sept. 11, 2008; accepted 0ct. 7, 2008.

This work was supported by the following Argentine institutions: Secretaría de Ciencia, Tecnología e Innovación Productiva, Fondo para la Investigación Científica y Tecnológica (PICT2004 05-26323), Universidad de Buenos Aires (UBACYT M050), and Consejo Nacional de Investigaciones Científicas y Técnicas (PIP 5890). M.G.M. was also supported by the J. S. Guggenheim Foundation. We thank Dr. Yukiori Goto (McGill University) for his advice about reverse microdialysis, Fernando Kasanetz for help with intracellular recordings in naive rats, and Carola Rodriguez (Universidad de Buenos Aires) for help with animal care.

Correspondence should be addressed to Pablo E. Pomata, Laboratorio de Fisiología de Circuitos Neuronales, Departamento de Fisiología y Biofísica, Facultad de Medicina, Universidad de Buenos Aires, Paraguay 2155, Ciudad de Buenos Aires (CP1121), Argentina. E-mail: neurofis@fmed.uba.ar.

D01:10.1523/JNEUROSCI.4343-08.2008

Copyright $\odot 2008$ Society for Neuroscience $\quad$ 0270-6474/08/2813384-06\$15.00/0
}

O’Donnell and Grace, 1995). Computational modeling and in vitro studies suggest that the NMDAR contributes to sustain the MSN UP state (Cepeda et al., 1993; Vergara et al., 2003; Wolf et al., 2005; Tseng et al., 2007). Moreover, excitatory amino acids induce plateaus and firing in the striatum in vivo via NMDAR selective effects (Herrling et al., 1983). However, the UP states recorded under ketamine anesthesia look similar to those seen under other anesthetics that lack of antagonist effects on NMDARs (Wilson and Kawaguchi, 1996; Mahon et al., 2001). Thus, although some studies suggest that NMDARs contribute to membrane depolarization and bistability in MSNs, direct in vivo evidence to support this view is lacking. Here, we assessed the contribution of striatal NMDARs to UP states and spontaneous firing in rat MSNs in vivo.

\section{Materials and Methods}

Adult male Sprague Dawley rats were used and cared for in accordance with institutional (CICUAL-RS2079/2007, UBA) and government (SENASA-RS617/2002, Argentina) regulations. All methods including signal analysis were similar to those described previously (Tseng et al., 2001; Kasanetz et al., 2006; Belluscio et al., 2007; Zold et al., 2007; Kasanetz et al., 2008). Recordings were performed under urethane anesthesia [1.2 $\mathrm{g} / \mathrm{kg}$ i.p. initially, then supplements as in the study by Kasanetz et al. (2006)]. Briefly, a concentric bipolar electrode was positioned in the deep layers of the motor cortex to record the local field potential (LFP; 0.1-300 $\mathrm{Hz}$ ) as described by Kasanetz et al. (2008). Then, a microdialysis probe (2 $\mathrm{mm}$ of exposed membrane; Bioanalytical Systems) was vertically inserted into the striatum $(+1.5 \mathrm{~mm}$ from bregma, $2.5-3 \mathrm{~mm}$ from midline, 5.5-6 mm below cortical surface) (Paxinos and Watson, 1997) and perfused at $2 \mu \mathrm{l} / \mathrm{min}$ with artificial CSF (aCSF). A precision switch with zero dead space allowed perfusing the cannula with either aCSF or aCSF containing drug. The composition of aCSF was (in mM): $147 \mathrm{NaCl}, 3 \mathrm{KCl}, 0.8$ $\mathrm{MgCl}, 1.2 \mathrm{CaCl}_{2}, 2.0 \mathrm{NaH}_{2} \mathrm{PO}_{4}, 2.0 \mathrm{Na}_{2} \mathrm{HPO}_{4}$; osmolarity: 290-300 $\mathrm{mOsm} / \mathrm{L}$; pH 7.4 (West et al., 2002). In different rats, intracellular or multiunit activity (MUA) was recorded from the dorsal striatum ipsilateral to the LFP recording, $0.5-1 \mathrm{~mm}$ caudal to the dialysis probe. For intracellular recordings, a glass electrode $(2 \mathrm{~m}$ potassium acetate and $1 \%$ 

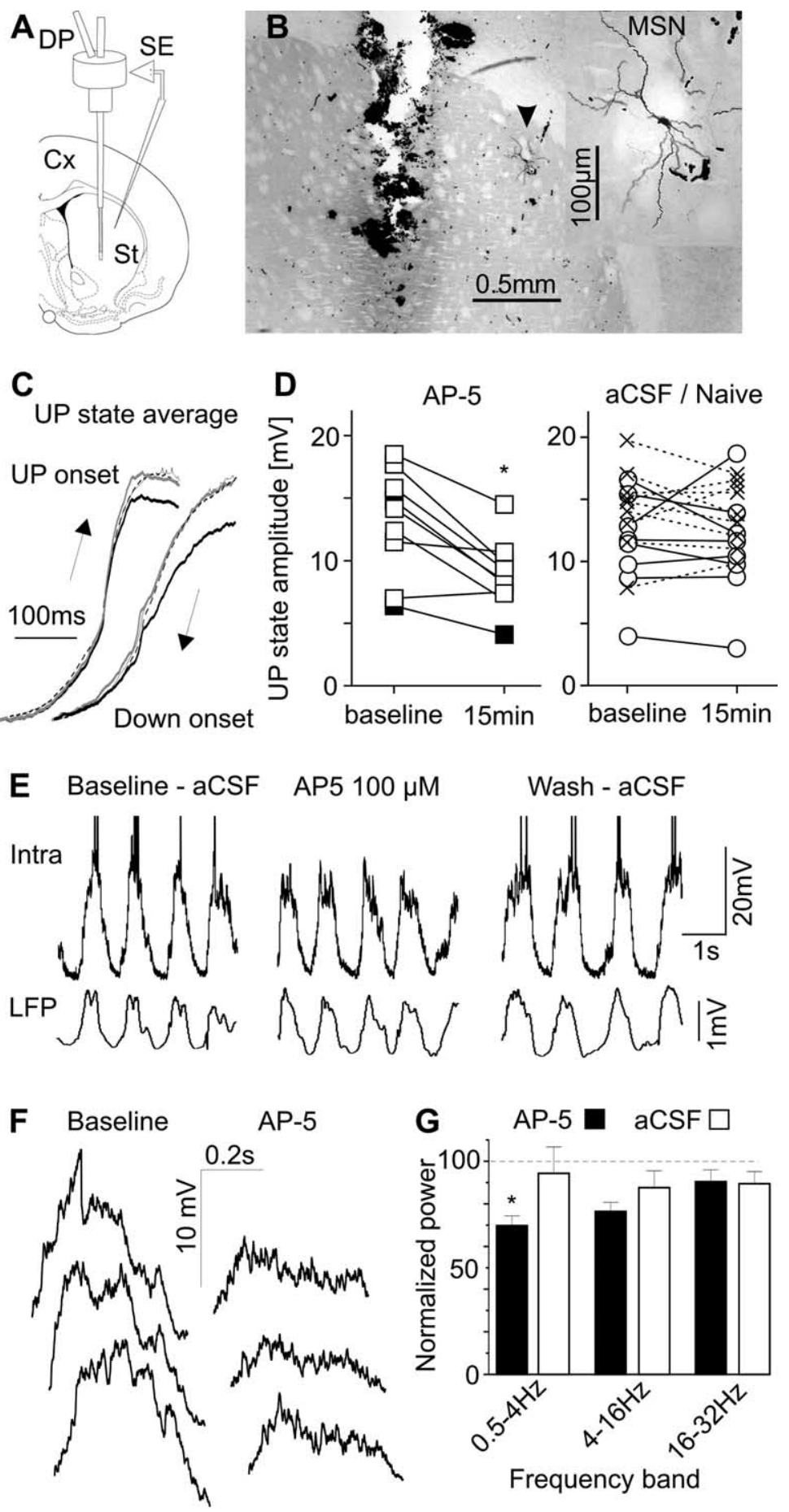

Figure 1. NMDAR contributes to MSN depolarization during UP states. $\boldsymbol{A}$, Diagram illustrating the position of microdialysis probes (DP) and sharp microelectrodes (SE). CX, Cerebral cortex; St, striatum. $\boldsymbol{B}$, Microphotograph showing the location of the probe and MSN (arrowhead) in a representative experiment. Inset, MSN at higher magnification. $C$, Averages of $>50$ UP state (left) and DOWN state (right) onsets recorded from a MSN in baseline conditions (thin lines), after 15 min of AP-5 $100 \mu \mathrm{m}$ infusion (black lines), and $40 \mathrm{~min}$ after discontinuing AP-5 (dashed lines). To allow comparing the slope of the waveforms, those corresponding to AP-5 were also scaled to the baseline maximum (gray lines). The time axis was inverted for transitions to the DOWN state, to allow assessing putative hysteretic effects of AP-5 (Wolf et al., 2005). Note that AP-5 reduced UP state amplitude without changing UP state slopes. D, Effect of AP-5 on UP state amplitude (left). AP- 5 was infused at $100 \mu \mathrm{m}$ in seven and at $200 \mu \mathrm{m}$ in two rats (filled squares). Right, Lack of change of UP state amplitude in eight MSNs recorded under continuous aCSF (circles) and nine MSNs recorded in naive rats (crosses). ${ }^{*} p<0.001$ versus baseline, Tukey test after $p<0.006$ interaction in a two-way ANOVA with MSN group and treatment (repeated measure) as factors. $\boldsymbol{E}$, Simultaneous cortical LFP and intracellular recording in one rat treated with AP-5 $100 \mu \mathrm{m}$. Spontaneous firing ceased during AP-5 administration. $\boldsymbol{F}$, Expanded view (500 ms after transition) of three consecutive UP states in the baseline condition and under AP-5 in another MSN. G, Frequency dependent effect of AP-5 on membrane potential during UP states. ${ }^{*} p<0.001$ versus baseline, Tukey's test after $p=0.003$ interaction in a two-way ANOVA. For technical details, see supplemental material (available at www.jneurosci.org).
Neurobiotin, 60-90 M $\Omega$ ) was lowered with an angle of $20^{\circ}$ in the coronal plane into the striatum (3-5 mm from brain surface). After cell penetration and complete removal of hyperpolarizing current, we examined the stability of the recordings for $2-5$ min before starting experimental protocols. MUA was recorded through a multielectrode (16-channels, $200 \mu \mathrm{m}$ vertical site spacing, $500 \mu \mathrm{m}$ horizontal shank spacing; NeuroNexus Technologies) positioned so as to yield a maximum of channels showing spontaneous spikes. After recording baseline activity during aCSF infusion, we studied the effect of competitive NMDAR antagonists on striatal activity [2-amino-5phosphono-pentanoic acid (AP-5), 100 or $200 \mu \mathrm{M}$; \pm 2 -amino-4-methyl-5-phosphono-3pentanoic acid ethyl ester (CGP39551), $20 \mu \mathrm{M}$ ]. Preliminary experiments showed that AP-5 at $100 \mu \mathrm{M}$ had no effect on MUA at $2 \mathrm{~mm}$ from the dialysis probe (data not shown). Considering that, because of diffusion, drug concentration at recordings sites was necessarily lower than in the probe, tissue concentrations of AP- 5 yielded in the present study should compare favorably with those producing NMDAR selective effects in vitro (Vergara et al., 2003; Pawlak and Kerr, 2008). Because striatal activity is modulated by global brain states (Kasanetz et al., 2002), all measures were performed on signals showing similar cortical slow wave activity, as assessed from LFP auto-spectra (5-30 min of baseline activity and 5-15 min under drug effect). Spike sorting was performed as described by Zold et al. (2007). Channels showing inconsistent spiking during the baseline period were not included in the analysis. The effects of NMDAR antagonism on the power of different frequency components of UP states was assessed as described in supplemental material, available at www.jneurosci.org. Probe positions were established postmortem. That of the multielectrode was determined by visualization of fluorescent material left along the electrode track (DiI, Invitrogen). Some MSNs were labeled with Neurobiotin, which was visualized through a peroxidase reaction.

\section{Results}

AP-5 reduces UP state amplitude

We recorded the UP states of MSNs simultaneously with the motor cortex LFP in urethane-anesthetized rats. The basic physiological properties of MSNs recorded in naive rats not implanted with microdialysis probes were compared with those of MSNs recorded at $<1 \mathrm{~mm}$ from a probe (Fig. $1 A, B$ ). No differences were observed in input resistance, UP state amplitude or duration, threshold, and action potential amplitude (Table 1), suggesting that the probe did not disturb the environment around the recording electrodes (West et al., 2002).

To determine whether NMDARs contribute to striatal UP states, we recorded the membrane potential of MSNs under 
aCSF for $10 \mathrm{~min}$ (baseline), and then for another 15-20 min under aCSF alone or aCSF containing the competitive NMDAR antagonist AP-5. AP-5 (100-200 $\mu \mathrm{M}, n=9)$ consistently reduced UP state amplitude from $13.2 \pm 1.4 \mathrm{mV}$ during baseline to $8.8 \pm$ $0.9 \mathrm{mV}$ (mean $\pm \mathrm{SEM}, p<0.05$ ) (Fig. $1 C, D$ ). In contrast, UP state amplitude did not change under continuous aCSF (baseline: $11.4 \pm 1.4 \mathrm{mV} ; 15 \mathrm{~min}$ later: $11.1 \pm 1.6 \mathrm{mV} ; n=8$ ) or in naive rats (baseline: $14.3 \pm 1.1 \mathrm{mV}$; $20 \mathrm{~min}$ later: $13.9 \pm 0.9 \mathrm{mV} ; n=9$ ) (Fig. $1 D$; supplemental material, available at www.jneurosci. org). Additionally, in two MSNs that fired spontaneously, AP-5 inhibited the firing. One of them could be recorded for long enough to washout AP-5 effects and showed complete recovery of its spontaneous firing (Fig. $1 \mathrm{E}$ ). Confirming the above findings, AP-5 reduced the power of the low frequency components of the membrane potential $(0.5-4 \mathrm{~Hz})$ without altering the fastest modulations occurring during UP states (Fig. $1 F$, G; supplemental material, available at www.jneurosci.org). In contrast, AP-5 had no effect on the slopes of UP states (Fig. 1C), UP state duration $(507 \pm 20$ and $505 \pm 11 \mathrm{~ms})$, and input resistance (40.3 \pm 8.6 and $41.0 \pm 12 \mathrm{M} \Omega$; baseline and AP-5, respectively). Thus, the data show a marked contribution of NMDARs to MSN depolarization during UP states.

\section{NMDAR antagonists reduce spontaneous firing of striatal ensembles}

To characterize NMDAR contribution to spontaneous firing in the striatum, we recorded the discharge activity of striatal ensembles through a multielectrode located at $0.5-1 \mathrm{~mm}$ from the dialysis probe in an additional group of rats (Fig. $2 A, B$ ). Striatal MUA was markedly modulated by slow waves. Most spikes occurred during the active part of slow waves (Fig. 2C), which in our deep LFP recordings has positive polarity (Fig. $1 E$ ) (Kasanetz et al., 2006). This is in agreement with published findings and indicates that most of the recorded spikes belonged to MSNs, because they only fire spontaneously during UP states (Wilson, 1993; Mahon et al., 2001; Kasanetz et al., 2002). We identified 72 channels with stable MUA along $30 \mathrm{~min}$ of baseline recording in nine rats, averaging $8.0 \pm 1.1$ channels per rat. In five rats (42 channels), AP-5 (100 $\mu \mathrm{M})$ visibly reduced spontaneous firing rate to $56 \%$ of baseline $(78.5 \pm 10.6$ versus $140 \pm 15$ spikes/min per channel, respectively, $p<0.05)$, without modifying the cortical LFP (baseline: $0.83 \pm 0.04 \mathrm{~Hz}, 53 \pm 1.6 \%$; AP-5: $0.80 \pm 0.03 \mathrm{~Hz}$, $54 \pm 2.2 \%$; peak frequency and relative power, respectively, as assessed from auto-spectra). Striatal activity showed complete recovery $40 \mathrm{~min}$ after discontinuing AP-5 (130.2 \pm 20 spikes/min per channel) (Fig. 2D,E). We also examined the effect of the competitive NMDAR antagonist CGP39551 $(20 \mu \mathrm{M})$ in four rats (30 channels). MUA dropped to $27.2 \%$ of baseline after $30 \mathrm{~min}$ of CGP39551 infusion $(p<0.05, n=4)$ (Fig. $2 F)$. No recovery was observed $1 \mathrm{~h}$ after discontinuing CGP39551 infusion.

In addition to MUA proceeding largely from MSNs, some channels exhibited spikes all over the slow wave (Fig. 2C), which could correspond to tonically active neurons (TANs). Supposedly, TANs are cholinergic interneurons and represent $<5 \%$ of the striatal neurons (Apicella, 2007). Because of intrinsic mechanisms, cholinergic interneurons exhibit tonic discharge (Bennett and Wilson, 1999). To determine whether the NMDAR modulates TANs in vivo, we performed spike sorting on MUA to separate spikes belonging to TANs from those belonging to neurons locked to the slow wave. We defined TANs by inspecting circular plots depicting spike occurrences as function of the phase angle of the slow wave. Seven neurons with roughly uniform circular distribution (i.e., similar probability of firing during ac-
Table 1. Electrophysiological properties of MSNs in rats with or without a microdialysis probe in the striatum

\begin{tabular}{lcc}
\hline Property & Dialysis probe $(n=17)$ & Naive $(n=9)$ \\
\hline Resting potential $(\mathrm{mV})$ & $-85.8 \pm 2.5$ & $-87.5 \pm 2.1$ \\
Input resistance $(\mathrm{M} \Omega)$ & $37.9 \pm 4.5$ & $47.4 \pm 9.1$ \\
Action potential threshold $(\mathrm{mV})$ & $-55.5 \pm 2.3$ & $-51.0 \pm 2.3$ \\
Action potential amplitude $(\mathrm{mV})$ & $52.5 \pm 2.2$ & $47.7 \pm 3$ \\
UP state duration (ms) & $504 \pm 14$ & $491 \pm 23$ \\
UP state amplitude $(\mathrm{mV})$ & $12.4 \pm 1.1$ & $14.3 \pm 1.1$ \\
\hline
\end{tabular}

Data are mean \pm SEM.

tive and silent parts of the wave) and firing rate $>2$ spikes/s were grouped as TANs (Fig. 3A-C). With comparative purposes, we also identified in neighboring channels 14 units that fired during the active part of slow waves. However, as we found difficult to sort reliably units firing at very low rates, we refrain from concluding that these spikes belonged exclusively to MSNs, which usually fire less than once per second. Moreover, some cholinergic interneurons and most fast spiking interneurons fire in consonance with slow waves (Reynolds et al., 2004; Mallet et al., 2005). Thus, these phasic units most likely represent a mixture of MSNs, fast spiking interneurons and cholinergic interneurons. AP-5 $(100 \mu \mathrm{M})$ reduced phasic units' discharge from $1.47 \pm$ $0.24-0.33 \pm 0.07$ spikes/s $(p<0.001)$, without affecting TANs located nearby (baseline: $3.74 \pm 0.34$ spikes/s, AP-5: $3.09 \pm 0.57$ spikes/s) (Fig. 3D,E). Thus, the analysis confirmed that NMDAR activation drives the spontaneous firing of MSNs and probably other phasically active striatal neurons.

\section{Discussion}

The present study demonstrates that striatal NMDARs contribute directly to drive MSNs to threshold during UP states. Previous studies have shown that exogenous excitatory amino acids induce plateaus and firing in striatal neurons in vivo (Herrling et al., 1983) and in vitro (Cepeda et al., 1993; Vergara et al., 2003) via NMDAR selective effects. However, this is the first study to show that striatal NMDAR activation by physiological signals gates information flow during UP states in the striatum in vivo.

It is accepted that cortical inputs induce the UP state in MSNs (Wilson, 1993; O'Donnell and Grace, 1995). Modeling studies have suggested that a sufficiently large depolarization initiated by AMPAR could remove $\mathrm{Mg}^{2+}$ block from the NMDA channel, allowing its synaptic activation during UP states (Wolf et al., 2005; Kepecs and Raghavachari, 2007). However, there is a debate as to how the NMDAR contributes to UP states. One biophysical model suggests that synaptic activation of a sufficiently large NMDA current can induce the UP state (Kepecs and Raghavachari, 2007). In agreement with this model, bath application of NMDA elicits recurrent plateaus in MSNs in slices. These plateaus can stand the blockade of AMPAR but not addition of AP-5 (Vergara et al., 2003). Our results showing that MSNs exhibit UP states despite local delivery of AP-5 are consistent with findings based on the systemic administration of noncompetitive NMDAR antagonists (Wilson and Kawaguchi, 1996; O'Donnell and Grace, 1998; Mahon et al., 2001) and do not support that NMDARs are necessary during UP state onset in vivo. A second model predicts that the NMDA current is delayed relative to the AMPA current during UP state onset (Wolf et al., 2005). This model MSN displays UP states even in complete absence of NMDA current, which are dependent on AMPAR synaptic input. Moreover, the main contribution of the NMDA current, because of its slow deactivation, is to UP state amplitude and firing, with increasing effects on the decay time to the DOWN state at high 
A
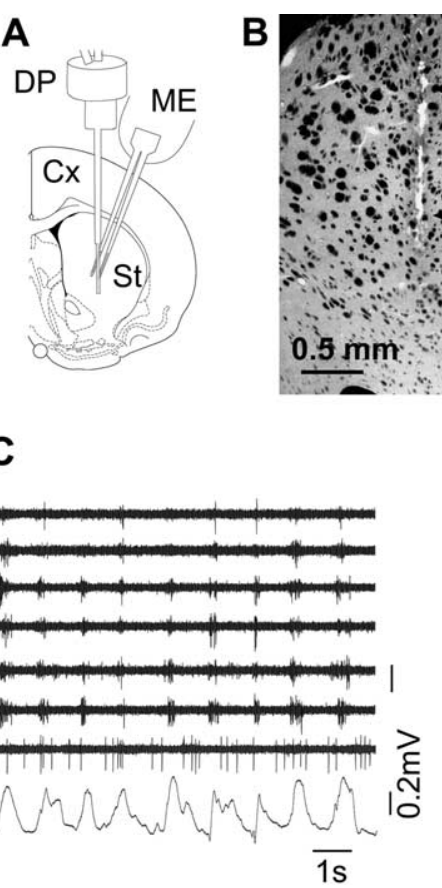

C
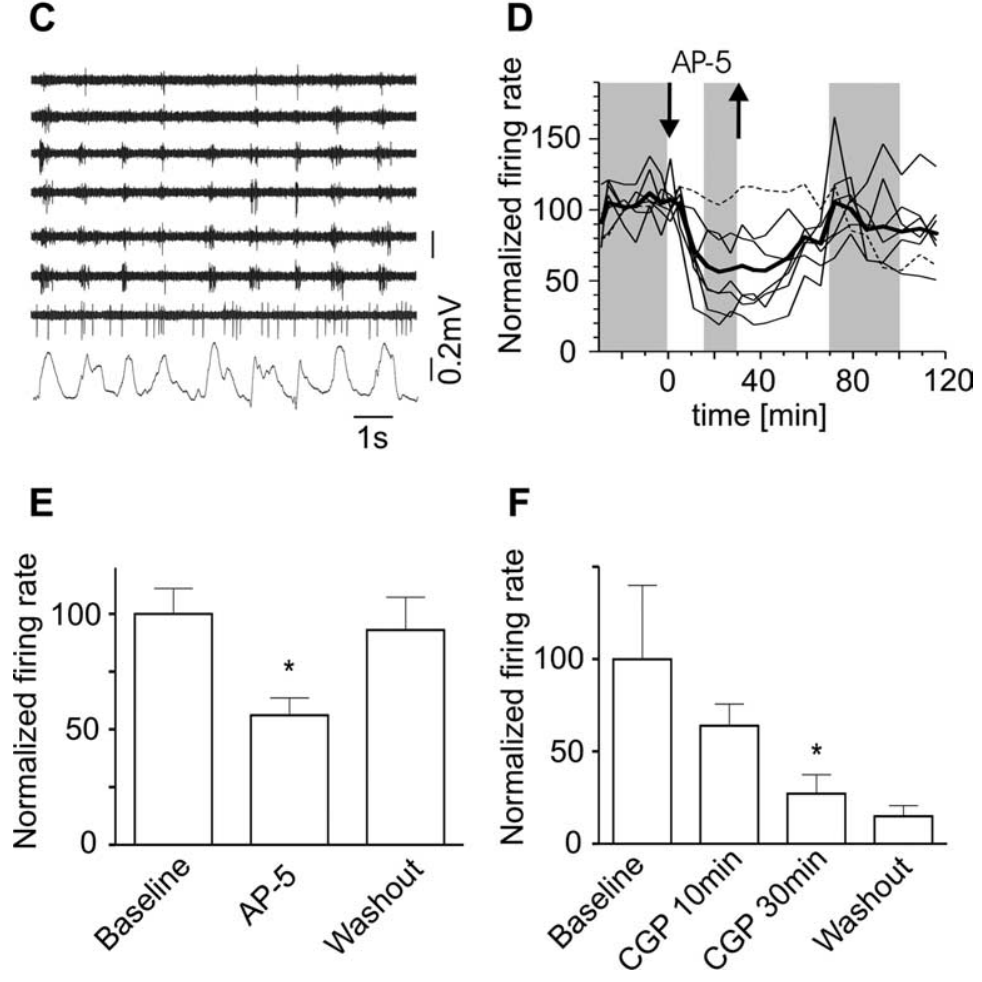

Figure 2. NMDAR-dependent striatal firing during slow waves. $\boldsymbol{A}$, Diagram illustrating the striatal location of the dialysis probe and multielectrode (ME). $\boldsymbol{B}$, Microphotographs showing the location of the microdialysis probe (left; transmitted light) and electrode shanks (right; Dil fluorescence in an adjacent section) in a representative experiment. C, Spontaneous activity recorded through seven striatal channels simultaneously with the frontal cortex LFP in a representative experiment (baseline). Note that most striatal neurons fired in relation with the slow wave (Kasanetz et al., 2006). The seventh channel shows tonic activity. D, Time course of striatal MUA during the same experiment, illustrating AP-5 effect (100 $\mu \mathrm{m})$. Each line represents a channel, except for the thick one, which is the average of all channels. The dashed line corresponds to the seventh channel in $C$. Within each channel, MUA was normalized to baseline discharge rate. The gray shading indicates epochs from which data were collected for the comparisons shown in $\boldsymbol{E}$. $\boldsymbol{E}$, Effect of AP-5 (100 $\mu \mathrm{m})$ on striatal MUA in five rats. $\boldsymbol{F}$, Effect of CGP39551 (20 $\mu \mathrm{m})$ in four rats. ${ }^{*} p<0.05$ versus baseline, Dunnett's test after repeated measures ANOVA; data are mean \pm SEM.

NMDA/AMPA ratios (Wolf et al., 2005). Our results are in general agreement with this model, but we could not confirm that NMDARs shape transition to the DOWN state. However, bath application of AP-5 shortens UP states in organotypic corticostriatal cultures (Tseng et al., 2007). Perhaps NMDARs have more influence on corticostriatal transmission in vitro, or the effects seen in vitro involve cortical NMDARs. Moreover, we found no contribution of NMDARs to the fastest membrane potential modulations occurring during UP states. Certainly, our findings fit better with the view that continued fast synaptic input rather than slow NMDAR currents outlasting an initial glutamatergic drive sustain MSN UP states in vivo.

The effects seen in MSNs in our study may involve other cellular elements of the striatum. In this sense, nitric oxide contributes to UP state amplitude in MSNs (West and Grace, 2004).
This, together with evidence showing that burst stimulation of the frontal cortex promotes nitric oxide production by striatal interneurons via NMDAR activation (Sammut et al., 2007), suggested that nitric oxide interneurons exert feedforward excitation on MSNs (Ondracek et al., 2008). However, nitric oxide effect relied on an increase in MSN input resistance, which is at odds with our findings. Our results are unlikely to be a result of NMDAR blockade in fast spiking interneurons, as this should have released MSNs from inhibition (Mallet et al., 2005). Last, we found that putative cholinergic interneurons showing tonic discharge during periods of low cortical input were insensitive to NMDAR antagonists. This is consistent with in vitro studies showing that cholinergic interneurons are less sensitive to NMDA than MSN (Cepeda et al., 2001). Although we cannot rule out a contribution of network mechanisms to the effects of NMDAR antagonists with this in vivo approach, the data show a marked NMDAR modulation of MSNs' spontaneous firing.

The functional implications of NMDAR gating of striatal firing remain speculative. As in other brain regions (Daw et al., 1993; Schiller and Schiller, 2001), striatal NMDARs could be involved in online information processing. A recent slice study shows that NMDA can induce coordinated sequences of UPs and burst firing in striatal assemblies, which could reflect pattern generation by striatal microcircuits (CarrilloReid et al., 2008). However, current studies often assume that striatal NMDARs are selectively recruited during learning to induce plasticity but do not contribute to performance (Atallah et al., 2007). Indeed, there is limited evidence that NMDARs support striatal firing in behaving animals (Sandstrom and Rebec, 2003). Although UP states have been reported in nonanesthetized rats (Wilson, 1993), our findings may be more relevant for information processing in slow wave sleep, because of its similarity with the brain state induced by urethane (Steriade et al., 2001; Mahon et al., 2006). Recent studies see slow waves as a global process, extending beyond the thalamocortical system to the hippocampus (Isomura et al., 2006) and striatum (Kasanetz et al., 2008), during which patterns of activity related to previous experience are replayed in all these structures (Wilson and McNaughton, 1994; Pennartz et al., 2004; Ribeiro et al., 2004). Moreover, reactivations of NMDARs over days or weeks could be necessary for memory consolidation (Wang et al., 2006). Thus, NMDAR gating of striatal firing could allow off-line processing of cortical input, supporting systems level consolidation (Dudai, 2004) and reinforcing changes in corticostriatal synapses that form part of a memory trace (Pawlak and Kerr, 2008). 
A

\section{Baseline firing rate}

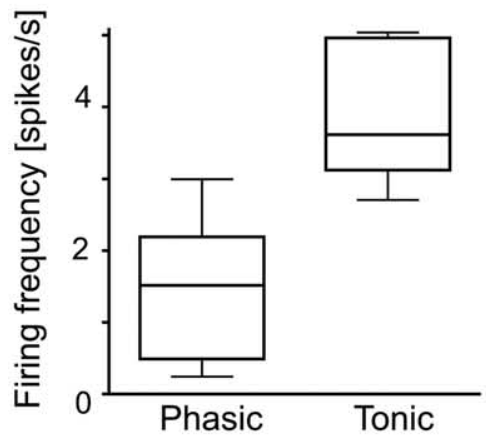

B

Phasic unit spikes

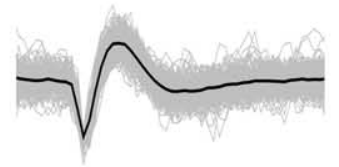

Tonic unit spikes

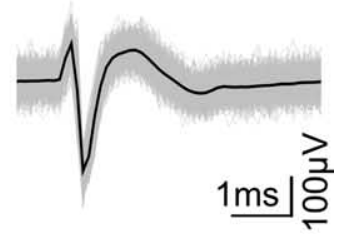

C
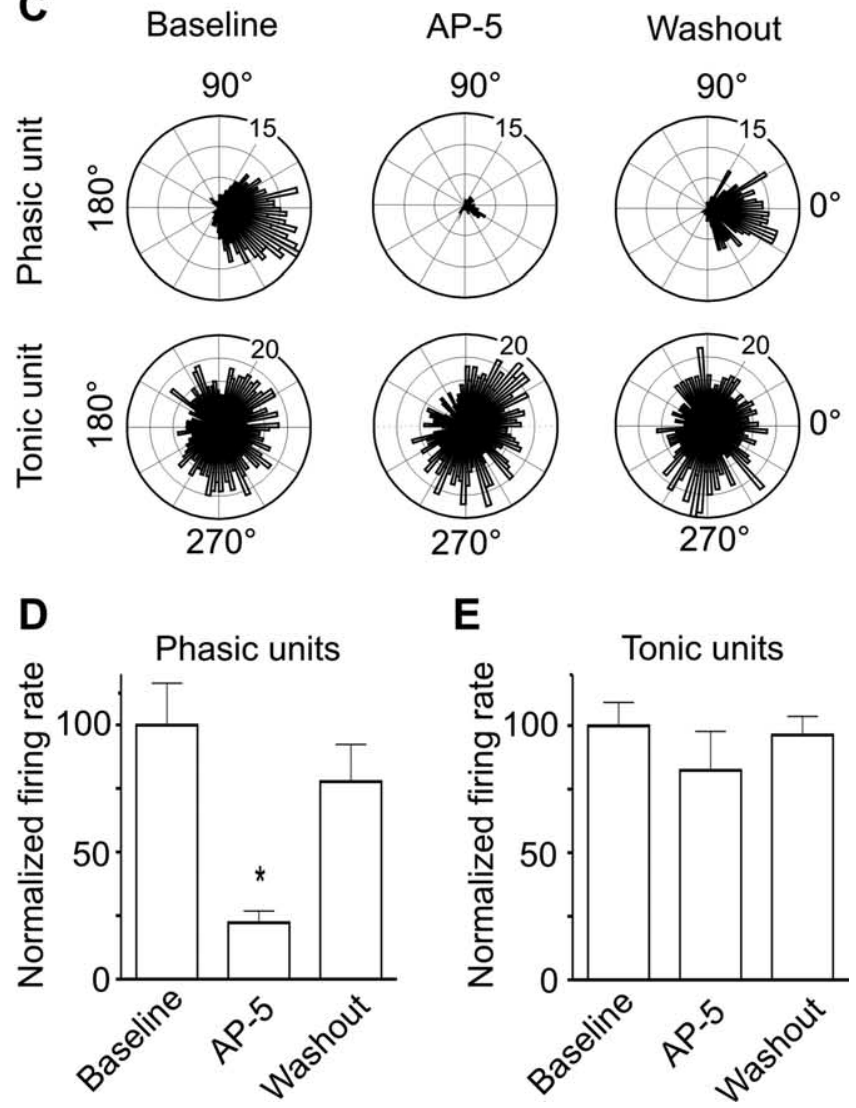

Figure 3. TANs' firing independent of NMDARs. $\boldsymbol{A}$, Firing rates of tonically $(n=7)$ and phasically $(n=14)$ active units in the baseline condition. Data are median, 25th-75th percentiles (box) and minimum and maximum values (whiskers). $\boldsymbol{B}$, Shape of spikes corresponding to representative phasic and tonic units. Black lines are the average of all collected spikes (in gray). C, Polar plots depicting discharge rate (radial axis) as function of slow wave angle (circular axis), corresponding to the phasically (above) and tonically (below) active units shown in $\boldsymbol{B}$. The maximum of the slow wave occurs at $0^{\circ}$. The TAN fired with similar probabilities in the left and right halves of the circle, suggesting that cortical input (which is high around $0^{\circ}$ ) had little influence on this unit. $\boldsymbol{D}, \boldsymbol{E}$, Effect of AP-5 on phasically and tonically active units. ${ }^{*} p<$ 0.01 versus baseline, Dunnett's test after repeated measures ANOVA; data are mean \pm SEM.

\section{References}

Apicella P (2007) Leading tonically active neurons of the striatum from reward detection to context recognition. Trends Neurosci 30:299-306.

Atallah HE, Lopez-Paniagua D, Rudy JW, O’Reilly RC (2007) Separate neural substrates for skill learning and performance in the ventral and dorsal striatum. Nat Neurosci 10:126-131.

Belluscio MA, Riquelme LA, Murer MG (2007) Striatal dysfunction in- creases basal ganglia output during motor cortex activation in parkinsonian rats. Eur J Neurosci 25:2791-2804.

Bennett BD, Wilson CJ (1999) Spontaneous activity of neostriatal cholinergic interneurons in vitro. J Neurosci 19:5586-5596.

Carrillo-Reid L, Tecuapetla F, Tapia D, Hernández-Cruz A, Galarraga E, Drucker-Colin R, Bargas J (2008) Encoding network states by striatal cell assemblies. J Neurophysiol 99:1435-1450.

Cepeda C, Buchwald NA, Levine MS (1993) Neuromodulatory actions of dopamine in the neostriatum are dependent upon the excitatory amino acid receptor subtypes activated. Proc Natl Acad Sci U S A 90:9576-9580.

Cepeda C, Itri JN, Flores-Hernández J, Hurst RS, Calvert CR, Levine MS (2001) Differential sensitivity of medium- and large-sized striatal neurons to NMDA but not kainate receptor activation in the rat. Eur J Neurosci 14:1577-1589.

Daw NW, Stein PS, Fox K (1993) The role of NMDA receptors in information processing. Annu Rev Neurosci 16:207-222.

Dudai Y (2004) The neurobiology of consolidations, or, how stable is the engram? Annu Rev Psychol 55:51-86.

Graybiel AM (1998) The basal ganglia and chunking of action repertoires. Neurobiol Learn Mem 70:119-136.

Herrling PL, Morris R, Salt TE (1983) Effects of excitatory amino acids and their antagonists on membrane and action potentials of cat caudate neurones. J Physiol 339:207-222.

Isomura Y, Sirota A, Ozen S, Montgomery S, Mizuseki K, Henze DA, Buzsáki G (2006) Integration and segregation of activity in entorhinalhippocampal subregions by neocortical slow oscillations. Neuron 52:871-882.

Kasanetz F, Riquelme LA, Murer MG (2002) Disruption of the two-state membrane potential of striatal neurones during cortical desynchronisation in anaesthetised rats. J Physiol 543:577-589.

Kasanetz F, Riquelme LA, O’Donnell P, Murer MG (2006) Turning off cortical ensembles stops striatal Up states and elicits phase perturbations in cortical and striatal slow oscillations in rat in vivo. J Physiol 577:97-113.

Kasanetz F, Riquelme LA, Della-Maggiore V, O'Donnell P, Murer MG (2008) Functional integration across a gradient of corticostriatal channels controls UP state transitions in the dorsal striatum. Proc Natl Acad Sci U S A 105:8124-8129.

Kelley AE, Andrzejewski ME, Baldwin AE, Hernandez PJ, Pratt WE (2003) Glutamate-mediated plasticity in corticostriatal networks: role in adaptive motor learning. Ann N Y Acad Sci 1003:159-168.

Kepecs A, Raghavachari S (2007) Gating information by two-state membrane potential fluctuations. J Neurophysiol 97:3015-3023.

Mahon S, Deniau JM, Charpier S (2001) Relationship between EEG potentials and intracellular activity of striatal and cortico-striatal neurons: an in vivo study under different anesthetics. Cereb Cortex 11:360-373.

Mahon S, Vautrelle N, Pezard L, Slaght SJ, Deniau JM, Chouvet G, Charpier S (2006) Distinct patterns of striatal medium spiny neuron activity during the natural sleep-wake cycle. J Neurosci 26:12587-12595.

Mallet N, Le Moine C, Charpier S, Gonon F (2005) Feedforward inhibition of projection neurons by fast-spiking GABA interneurons in the rat striatum in vivo. J Neurosci 25:3857-3869.

O’Donnell P, Grace AA (1995) Synaptic interactions among excitatory afferents to nucleus accumbens neurons: hippocampal gating of prefrontal cortical input. J Neurosci 15:3622-3639.

O'Donnell P, Grace AA (1998) Phencyclidine interferes with the hippocampal gating of nucleus accumbens neuronal activity in vivo. Neuroscience $87: 823-830$.

Ondracek JM, Dec A, Hoque KE, Lim SA, Rasouli G, Indorkar RP, Linardakis J, Klika B, Mukherji SJ, Burnazi M, Threlfell S, Sammut S, West AR (2008) Feed-forward excitation of striatal neuron activity by frontal cortical activation of nitric oxide signaling in vivo. Eur J Neurosci 27:1739-1754.

Pawlak V, Kerr JN (2008) Dopamine receptor activation is required for corticostriatal spike-timing-dependent plasticity. J Neurosci 28:2435-2446.

Paxinos G, Watson C (1997) The rat brain in stereotaxic coordinates. San Diego: Academic.

Pennartz CM, Lee E, Verheul J, Lipa P, Barnes CA, McNaughton BL (2004) The ventral striatum in off-line processing: ensemble reactivation during sleep and modulation by hippocampal ripples. J Neurosci 24:6446-6456.

Reynolds JN, Hyland BI, Wickens JR (2004) Modulation of an afterhyperpolarization by the substantia nigra induces pauses in the tonic firing of striatal cholinergic interneurons. J Neurosci 24:9870-9877.

Ribeiro S, Gervasoni D, Soares ES, Zhou Y, Lin SC, Pantoja J, Lavine M, 
Nicolelis MA (2004) Long-lasting novelty-induced neuronal reverberation during slow-wave sleep in multiple forebrain areas. PLoS Biol 2:E24.

Sammut S, Park DJ, West AR (2007) Frontal cortical afferents facilitate striatal nitric oxide transmission in vivo via a NMDA receptor and neuronal NOS-dependent mechanism. J Neurochem 103:1145-1156.

Sandstrom MI, Rebec GV (2003) Characterization of striatal activity in conscious rats: contribution of NMDA and AMPA/kainate receptors to both spontaneous and glutamate-driven firing. Synapse 47:91-100.

Schiller J, Schiller Y (2001) NMDA receptor-mediated dendritic spikes and coincident signal amplification. Curr Opin Neurobiol 11:343-348.

Steriade M, Timofeev I, Grenier F (2001) Natural waking and sleep states: a view from inside neocortical neurons. J Neurophysiol 85:1969-1985.

Tseng KY, Kasanetz F, Kargieman L, Riquelme LA, Murer MG (2001) Cortical slow oscillatory activity is reflected in the membrane potential and spike trains of striatal neurons in rats with chronic nigrostriatal lesions. J Neurosci 21:6430-6439.

Tseng KY, Snyder-Keller A, O’Donnell P (2007) Dopaminergic modulation of striatal plateau depolarizations in corticostriatal organotypic cocultures. Psychopharmacology 191:627-640.

Vergara R, Rick C, Hernández-López S, Laville JA, Guzman JN, Galarraga E, Surmeier DJ, Bargas J (2003) Spontaneous voltage oscillations in striatal projection neurons in a rat corticostriatal slice. J Physiol 553:169-182.
Wang H, Hu Y, Tsien JZ (2006) Molecular and systems mechanisms of memory consolidation and storage. Prog Neurobiol 79:123-135.

West AR, Grace AA (2004) The nitric oxide-guanylyl cyclase signaling pathway modulates membrane activity states and electrophysiological properties of striatal medium spiny neurons recorded in vivo. J Neurosci 24:1924-1935.

West AR, Moore H, Grace AA (2002) Direct examination of local regulation of membrane activity in striatal and prefrontal cortical neurons in vivo using simultaneous intracellular recording and microdialysis. J Pharmacol Exp Ther 301:867-877.

Wilson CJ (1993) The generation of natural firing patterns in neostriatal neurons. Prog Brain Res 99:277-297.

Wilson CJ, Kawaguchi Y (1996) The origins of two-state spontaneous membrane potential fluctuations of neostriatal spiny neurons. J Neurosci 16:2397-2410.

Wilson MA, McNaughton BL (1994) Reactivation of hippocampal ensemble memories during sleep. Science 265:676-679.

Wolf JA, Moyer JT, Lazarewicz MT, Contreras D, Benoit-Marand M, O’Donnell P, Finkel LH (2005) NMDA/AMPA ratio impacts state transitions and entrainment to oscillations in a computational model of the nucleus accumbens medium spiny projection neuron. J Neurosci 25:9080-9095.

Zold CL, Ballion B, Riquelme LA, Gonon F, Murer MG (2007) Nigrostriatal lesion induces D2-modulated phase-locked activity in the basal ganglia of rats. Eur J Neurosci 25:2131-2144. 